\title{
Evaluation of two passive samplers for the analysis of organophosphate esters in the ambient air
}

\author{
Ruirui Liu ${ }^{\mathrm{a}, \mathrm{b}}$, Yongfeng Lin ${ }^{\mathrm{a}}$, Runzeng Liu ${ }^{\mathrm{a}}$, Fanbao Hu ${ }^{\mathrm{a}}$, Ting Ruan ${ }^{\mathrm{a}, *}$, Guibin Jiang a,b \\ a State Key Laboratory of Environmental Chemistry and Ecotoxicology, Research Center for Eco-Environmental Sciences, Chinese Academy of Sciences, \\ Beijing 100085, China \\ ${ }^{\mathrm{b}}$ Environment Research Institute, Shandong University, Jinan 250100, China
}

\section{A R T I C L E I N F O}

Article history:

Received 29 July 2015

Received in revised form

8 September 2015

Accepted 12 September 2015

Available online 16 September 2015

Keywords:

Organophosphate esters

Passive air sampler

Polyurethane foam

Sorbent-impregnated polyurethane foam

\begin{abstract}
A B S T R A C T
Both polyurethane foam (PUF) and sorbent-impregnated PUF (SIP) passive air sampling (PAS) methods were deployed and compared separately for the analysis of organophosphate esters (OPEs) in outdoor atmospheric environment. During an continuous period of 84 days, parallel samples were also collected by a high-volume active air sampler (HV-AAS) to assess the contamination levels and to calibrate uptake parameters of PAS. The total concentration of OPEs in both particulate and gaseous phases ranged from 1.50 to $5.64 \mathrm{ng} \mathrm{m}^{-3}$ in ambient air. Tris(2-chloroisopropyl) phosphate (TCPP) was the dominating analog, representing $78 \pm 9 \%$ of total OPE concentration. SIP-PAS showed longer linear-phase sampling period for TCPP, and accumulated more amount of the most volatile triethyl phosphate (TEP) and tributyl phosphate (TBP) homologues, while similar sorption performances of both PAS methods were found for most of the semi-volatile OPEs. Linear sampling rates in PUF-PAS and SIP-PAS disks were calculated for individual OPEs except for TEP and TBP, and the average uptake rates $\left(3.3 \pm 1.1\right.$ and $3.5 \pm 1.7 \mathrm{~m}^{3} \mathrm{~d}^{-1}, \mathrm{re}^{-}$ spectively) were close to the acknowledged value $\left(4 \mathrm{~m}^{-3} \mathrm{~d}^{-1}\right)$ for persistent organic pollutants. Besides, isotopic labeled $\mathrm{D}_{15}$-Triphenyl phosphate (TPhP) could be used as a viable depuration compound to calculate site-specific sampling rates of OPEs, with a linear loss of up to $~ 60 \%$ at the end of deployment time.
\end{abstract}

(c) 2015 Elsevier B.V. All rights reserved.

\section{Introduction}

Organophosphate esters (OPEs) are anthropogenic industrial derivates of phosphoric acid, which are substituted by a combination of alkyl chains, partly chlorinated alkyl chains and aromatic functional groups [1]. Chlorinated OPEs are mainly used as flame retardants (FRs), while the non-halogenated ones have additional uses, such as plasticizers and antifoaming agents, hydraulic fluids, and electronics [2,3]. Environmental measurements of OPEs could date back to the 1970s [4]. Nevertheless, increasing awareness has been focused on the chemicals due to their usage as alternative FRs for polybrominated diphenyl ethers (PBDEs) [5]. The global demands of OPEs are expected to increase and the resulting more production and emissions into the environment may cause reemerging concern [6,7]. An overview discussed the environmental levels of FRs in several monitoring studies [8], indicating that total OPE concentrations generally exceeded those of PBDEs in indoor air environment, and the human exposure potentials caused by

\footnotetext{
* Corresponding author. Fax: +861062849179.

E-mail address: tingruan@rcees.ac.cn (T. Ruan).
}

OPE contamination appeared to be higher.

OPEs are generally recognized as semi-volatile additives [9], which could be released into the environment from commercial products during volatilization, abrasion and dissolution [2]. Adverse health impacts, such as carcinogenic, neurotoxic, reproductive and hemolytic effects [8,10-14], have also been observed in biological tests. Hydrophobic OPE analogues, especially the chlorinated ones, are relatively stable toward biodegradation and might have persistent and long range transport abilities [15]. OPEs have been found globally in various environmental matrices $[1,5,15,16]$, especially the indoor and outdoor environment. Simultaneous air sampling is beneficial to assessing the sources and fates of OPEs through spatial mapping studies. For instance, in order to investigate the occupational exposure to eight organophosphorus compounds, low volume active air samplers were utilized at electronics dismantling facilities and social premises, with total OPE concentrations quantified in the range of 90-3800 $\mathrm{ng} \mathrm{m}^{-3}$ [16]. High-volume active air samplers (HV-AAS) were also applied to investigate organophosphorus compounds in airborne particles from Indian, Arctic, Pacific and Southern Ocean. The sum of OPEs ranged from $120 \mathrm{pg} \mathrm{m}^{-3}$ to $2900 \mathrm{pg} \mathrm{m}^{-3}$, and 
the air concentrations decreased sharply from continents toward the open oceans [1].

Active air sampling techniques, such as HV-AAS, could offer information on gas and particle phase distribution in high temporal resolution, however, it is not suitable for massive field monitoring due to the dependence on power supplies as well as instrumental expenses [17]. Nevertheless, passive air samplers (PAS) could provide integrated data on a long sampling period with simplicity and low cost [18], making it feasible to simultaneous sampling at a large geographical scale. Since 2005, a Global Atmospheric Passive Sampling study was performed for detecting persistent organic pollutants (POPs) both spatially and temporally $[19,20]$. Polyurethane foam (PUF) disk is particularly used as the passive sampling device because of its excellent performance on various kinds of semi-volatile contaminants over periods of several weeks [21]. Shoeib et al. further developed a novel sorbentimpregnated polyurethane foam (SIP) technique, in which XAD adsorbent powder is impregnated onto the macroreticular surface of traditional PUF disk [22]. Compared with PUF, SIP led to higher sorption capacity and longer linear-phase distribution period for more volatile chemicals [17]. Both PUF-PAS and SIP-PAS disks were deployed for measuring polychlorinated biphenyls and neutral polyfluoroalkyl compounds [23], which indicated that SIP-PAS disks had promising sorption capacities for the measurement of both legacy and emerging POPs.

For air sampling of OPEs in indoor air and remote marine atmosphere, active air samplers were generally applied in most previously reported investigations $[1,5,24,25]$. While, few techniques were tested for passive sampling of OPEs. A passive flux sampler containing an Empore C18FF adsorbent disk and a circular glass plate was developed for the measurement of OPE emissions from building materials in indoor environment [26]. Nevertheless, specific usages of the technique might limit its application to ambient air samples. In this work, both active HV-AAS and passive (PUF-PAS and SIP-PAS) techniques were used for ambient air sampling. The specific objectives include (i) to monitor the contamination levels and compositions of OPEs in outdoor environment, (ii) to establish a robust passive sampling method for OPEs, (iii) to assess the sorption comparability of two PAS disks.

\section{Experimental}

\subsection{Materials}

Tri(2-chloroethyl) phosphate (TCEP), Tri-n-propylphosphate (TnPP), Tris(2-chloroisopropyl) phosphate (TCPP), Triethyl phosphate (TEP), Tris(2-chloro-1-(chloromethyl)ethyl) phosphate (TDCP), Tributyl phosphate (TBP), Triphenyl phosphate (TPhP), Cresyl Diphenyl Phosphate (CDP), Tri-3-cresyl phosphate (TCrP) were purchased from Dr. Ehrenstorfer GmbH (Wesel, Germany). $\mathrm{D}_{15}$-TEP (98\%), $\mathrm{D}_{27}$-TBP (98\%) and $\mathrm{D}_{15}$-TPhP (98\%) were from Cambridge Isotope Laboratories (Tewksbury, MA). High-performance liquid chromatography (HPLC) grade hexane, dichloromethane, acetone, methanol and acetonitrile were purchased from JT Baker (Phillipsburg, NJ). Ultrapure water was prepared using a Milli-Q system (Millipore, Billerica, MA).

\subsection{Sampling}

Before sampling, PUF disks $(14 \mathrm{~cm}$ diameter $\times 1.35 \mathrm{~cm}$ thick, surface area $365 \mathrm{~cm}^{2}$, mass $4.40 \mathrm{~g}$, volume $207 \mathrm{~cm}^{3}$, Tisch Environmental, Cleves, $\mathrm{OH}$ ) were pre-washed by hexane/dichloromethane $(1 / 1, v / v)$ using accelerated solvent extraction system (ASE 350, Dionex Corporation). SIP disks were prepared by impregnating PUF with finely grounded XAD-4 resin $(\sim 0.75 \mu \mathrm{m}$,
Supelco, Bellefonte, PA), following the method reported by Shoeib et al. [22]. After that, each of $20.0 \mathrm{ng} \mathrm{D}_{27}$-TBP and $\mathrm{D}_{15}$-TPhP was spiked onto both PAS disks as depuration compounds (DCs). Then, PUF and SIP disks were individually placed inside laboratory-made stainless steel chambers to preserve a low-wind sampling environment $[27,28]$. The constructions of stainless steel chambers were mentioned elsewhere [22,27]. Both eleven PUF and SIP disks were deployed approximately two meters above the roof on top of the laboratory building $\left(40^{\circ} 00^{\prime} 26.94^{\prime \prime} \mathrm{N}, 116^{\circ} 20^{\prime} 15.10^{\prime \prime} \mathrm{E}\right)$ in our research center, with a total height of approximately $14 \mathrm{~m}$. The disks were harvested one at a time on every Friday in the sampling period from July 11 to September 26, 2014.

Parallel samples were also collected by using one HV-AAS sampler, which was located $10 \mathrm{~m}$ away from the PAS devices. It operated on every Tuesday and Friday from July 11 to September 30. The sampling rate was $500 \mathrm{~L} / \mathrm{min}$, and a total air volume of $600 \mathrm{~m}^{3}$ was collected. In order to trap OPEs from airborne particles, a glass fiber filter (GFF, $10.16 \mathrm{~cm}$ diameter, Whatman, Piscataway, NJ) was placed in front of a glass cartridge packed with two PUF (76 mm diameter $\times 60 \mathrm{~mm}$ thick) stacks for gas-phase analytes. All samples were collected, sealed with aluminum foil and stored at $-20^{\circ} \mathrm{C}$ until analysis.

\subsection{Sample pretreatment and instrumental analysis}

The GFFs, PUF stacks, PUF-PAS and SIP-PAS disks were pretreated separately. Prior to extraction, each active sample (GFFs and PUF stacks) was spiked with $20.0 \mathrm{ng} \mathrm{D}_{15}$-TPhP as surrogate standard. While, no additional isotopic-labeled standards were added for passive samples, and $\mathrm{D}_{27}-\mathrm{TBP}$ and $\mathrm{D}_{15}$-TPhP were tested as depuration compounds. All samples had the same extraction and purification procedures. In brief, hexane/dichloromethane (1/ $1, \mathrm{v} / \mathrm{v}$ ) was used as the extraction solvent. Accelerated solvent extraction was performed at $100{ }^{\circ} \mathrm{C}$ and 1500 psi in three static cycles of $10 \mathrm{~min}$. The extracts were concentrated to $\sim 2 \mathrm{~mL}$ by rotary evaporation and transferred to a gel permeation chromatography column (GPC, $400 \times 30 \mathrm{~mm}$, i.d.) packed with Biobeads S-X3 (200-400 mesh, Bio-Rad Laboratories, Hercules, CA). A hexane/dichloromethane mixture $(1 / 1, v / v)$ was used as eluent and the second fraction of $120 \mathrm{~mL}$ eluent was collected after discarding the first $110 \mathrm{~mL}$ fraction. The solvents were then concentrated to $\sim 2 \mathrm{~mL}$, and passed through an $8 \mathrm{~g}$ neutral alumina column (6-325 mesh, Sigma-Aldrich, St. Louis, MO), which was activated at $600^{\circ} \mathrm{C}$ for $6 \mathrm{~h}$ and 5\% water-deactivated before use. The column was preconditioned using $30 \mathrm{~mL}$ of hexane, and all target analytes were eluted with $80 \mathrm{~mL}$ of 1:3 hexane/dichloromethane mixture $(\mathrm{v} / \mathrm{v})$. Finally, the eluate was concentrated to $1 \mathrm{~mL}$, transferred into LC vials, and solvent exchanged with $980 \mu \mathrm{l}$ of $6: 4$ acetonitrile/ water mixture $(\mathrm{v} / \mathrm{v})$. Twenty microliters of $\mathrm{D}_{15}$-TEP $(10.0 \mathrm{ng})$ was further added into each vial as injection standard.

Analyte quantification was performed on an API 5500 triplequadrupole mass spectrometer (AB SCIEX Inc. Framingham, MA) interfaced with an Ultimate 3000 ultrahigh performance liquid chromatograph (Thermo Fisher Scientific Inc., Waltham. MA). An Acquity UPLC BEH C8 analyte column $(2.1 \mathrm{~mm}$ i.d. $\times 100 \mathrm{~mm}$ length, $1.7 \mu \mathrm{m}$, Waters) was selected for analyte separation. Column temperature was set as $45^{\circ} \mathrm{C}$. Acetonitrile and water were used as mobile phases, and the flow rate was $0.2 \mathrm{~mL} / \mathrm{min}$. Electrospray ionization (ESI) source was employed in positive ion mode. Curtain gas and collision gas were set as 35 and 7 psi, respectively. Ionspray voltage was $5500 \mathrm{v}$, and ionspray temperature was $450^{\circ} \mathrm{C}$. The flow gradient program as well as confirmation and quantification ions for each analyte in multiple reaction monitoring mode were shown in Tables S1 and S2. 


\subsection{Quality assurance and quality control}

Before loading samples, all extraction cells of ASE system were pre-rinsed with hexane/dichloromethane $(1 / 1, v / v)$ to prevent cross-contamination. The recoveries of the matrix-spiked samples (mean \pm standard deviation, 10.0 ng of native homologues) ranged from $72 \pm 8 \%$ to $92 \pm 13 \%$. Surrogate standards were also used to indicate recoveries during the analysis of samples and the values were $109 \pm 30 \%, 110 \pm 33 \%, 117 \pm 23 \%$ for the PUF stacks, GFFs and blank PAS disks. Besides, in order to assess matrix effects in the samples, $\mathrm{D}_{15}$-TEP (10.0 ng) was spiked as the injection standard. The average response values were $66 \%$ and $74 \%$ for gas and particle phase in HV-AAS samples, while $98 \%$ and $93 \%$ were found for PUFPAS and SIP-PAS disks, respectively. In order to check potential contamination in pretreatment procedures, three blanks were treated along with each batch of six air samples. The residue levels in the blanks were not quantifiable, except for TDCP $(<11 \%$ in the same batch of air samples). Thus, concentrations of target compounds in the samples were calibrated by eliminating the contamination of blanks and were corrected by recoveries of surrogate standards.

The signal-to-noise $(\mathrm{S} / \mathrm{N})$ ratios of ten was defined as the limit of quantification (LOQ). Individually, the LOQs were in the range of $0.13 \mathrm{pg}$ (TnPP) to $60.6 \mathrm{pg}$ (TCPP). Eight concentration points were used in the calibration curve and the gradient was $0.5,1,2,5,10$, $20,50,100 \mathrm{ng} \mathrm{mL}^{-1}$, with the regression coefficients $\left(R^{2}\right)$ higher than 0.99. For analyte with concentration exceeded the linear dynamic range, 10-fold dilution was performed by acetonitrile/ water mixture containing the same level $\left(10.0 \mathrm{ng} \mathrm{mL}^{-1}\right)$ of injection standard. Breakthrough experiments were also performed to check the sampling efficiency of PUF stacks for trapping gaseous compounds. Six upper PUF disks and corresponding bottom ones were randomly selected and analyzed separately. TDCP was not observed in both PUF stacks. While, $>95 \%$ of the other gaseous target analytes accumulated on the upper PUF stack disks, indicating that breakthrough did not occur in the sampling procedure.

\subsection{Theory of PAS}

The uptake mechanism of passive air sampling has been stated in previous studies $[17,22,28]$. In brief, during sampling period when air flows through the chamber and chemicals accumulate onto sampling media, passive sampling medium-air partition coefficient $\left(K_{\mathrm{PSM}-\mathrm{A}}\right)$ and air-side mass-transfer coefficient $\left(k_{\mathrm{A}}\right.$, $\mathrm{cm} \mathrm{d}^{-1}$ ) govern the analyte uptake profile. In this study, the profile can be described by Eq. (1).

$C_{\mathrm{PSM}}=K_{\mathrm{PSM}}-A \times C_{\mathrm{A}} \times\left\{1-\exp -\left[\left(\frac{A_{\mathrm{PSM}}}{V_{\mathrm{PSM}}} \times \frac{k_{\mathrm{A}}}{K_{\mathrm{PSM}}-A}\right) \times t\right]\right\}$

where $C_{\mathrm{PSM}}$ and $C_{\mathrm{A}}$ are the analyte concentrations $\left(\mathrm{pg} \mathrm{m}^{-3}\right)$ in the passive sampling medium (PSM) and air, $A_{\mathrm{PSM}}$ and $V_{\mathrm{PSM}}$ are planar area $\left(370 \mathrm{~cm}^{2}\right)$ and volume $\left(210 \mathrm{~cm}^{3}\right)$ of the PSM, $t$ is the employed time (days). $C_{\mathrm{A}}$ was the total analyte concentration measured by HV-AAS in both gas and particle phases, as airborne and particle-associated contaminants could possibly be scavenged by PSM.

The uptake profile between PAS and air could be divided into three stages. Initially, the amount of analyte in PSM is few, which thus increases linearly. This is a kinetically controlled linear stage. As analytes build up in PSM, uptake rate is reduced. The sampling process goes through curvilinear stage, and finally $C_{\mathrm{PSM}}$ establishes equilibrium (equal fugacity).

As shown in Eq. (1), uptake is a function of $k_{\mathrm{A}}, A_{\mathrm{PSM}}$ and $C_{\mathrm{A}}$ in the linear stage and the initial slope, $b$, is equal to $k_{\mathrm{A}} A_{\mathrm{PSM}} C_{\mathrm{A}}$. The sampling rate $\left(R, \mathrm{~m}^{3} \mathrm{~d}^{-1}\right)$, an important parameter used to assess the volume of air sampled over time, could be derived by dividing the slope $\mathrm{b}$ by $C_{\mathrm{A}}$ in the linear phase.

Besides, $K_{\text {PSM-A }}$ could indicate the extent to which analytes are collected relative to air on the PSM, and vary according to the type of PUF disk as well as the amount of XAD-4 impregnated. It could be described as the equivalent volume of air $\left(V_{\mathrm{AIR}}\right)$ which contains the same amount of chemical as 1 unit volume of PAS under equilibrium conditions, as well as the ratio of $c_{\mathrm{PSM}}$ divided by $c_{\mathrm{A}}$ [28].

$K_{\mathrm{PSM}-\mathrm{A}}=V_{\mathrm{AIR}} / V_{\mathrm{PSM}}=c_{\mathrm{PSM}} / c_{\mathrm{A}}$

In addition, the uptake profile could also be influenced by the chamber housing design as well as meteorological factors including temperature and wind speed $[17,22,28,29]$.

\section{Result and discussion}

\subsection{Atmospheric OPE concentrations by active sampler}

In this work, HV-AAS samples were used to monitor the OPE airborne contamination levels as well as to calculate averaged concentrations of each target analyte for passive sampling parameter calibration (Table S3). Except for TnPP, all other OPE compounds were positively identified in the ambient air samples. TCPP was the predominant analog with averaged proportions in the range of $78 \pm 9 \%$ in total OPE concentrations ( $\sum$ OPEs, Fig. 1 ). This was followed by TCEP and TPhP, which contributed to $11 \pm 8 \%$ and $6 \pm 4 \%$ in $\sum$ OPEs. Other target analytes had even less contributions $(<5 \%)$ in OPE concentration compositions, probably showing their limited applications in China. The ambient air compositions of OPE chemicals were extensively studied in many countries. The survey in the atmosphere of Mediterranean and Black Seas [5] showed that TCPP contributed $42 \pm 10 \%$ in the total OPE concentrations, with TnBP and TCEP representing $14 \pm 5 \%$ and $15 \pm 8 \%$ subsequently. In North Sea atmosphere of Germany [30], TCPP was also the most abundant OPE with a proportion of $60 \pm 16 \%$. Yang measured the particle size-specific distributions of OPEs in office

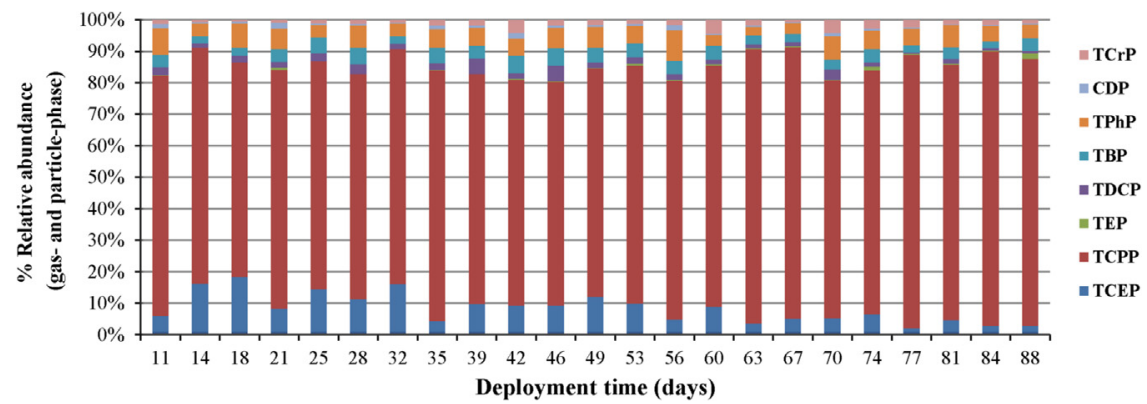

Fig. 1. Composition profiles of target OPEs in total air concentration (sum of gas- and particle-phase) measured by HV-AAS. 
air [24], and found that chlorinated OPEs (TCPP, TCEP, TDCPP) accounted for $69-99 \%$ of total OPEs. Nevertheless, another study showed that TCEP was the predominant OPE compound $(45 \pm 12 \%)$, which was followed by TCPP $(29 \pm 9 \%)$ in atmospheric particles over the northern South China Sea [25]. It may be resulted from the continuous use of TCEP near the deployed places, while TCEP is gradually replaced due to the higher toxic effects [8].

The total concentration (in both particulate and gaseous phases) ranged from 1.50 to $5.64 \mathrm{ng} \mathrm{m}^{-3}$ (mean concentration: $3.25 \mathrm{ng} \mathrm{m}^{-3}$ ), while individual contamination levels of TCPP, TCEP, TPhP, TBP, TDCP, TCrP, CDP and TEP ranged from 1.07-4.92 $\mathrm{ng} \mathrm{m}^{-3}$, $70.1-697 \mathrm{pg} \mathrm{m}^{-3}, 81.3-292 \mathrm{pg} \mathrm{m}^{-3}, 76.6-235 \mathrm{pg} \mathrm{m}^{-3}, 19.2-126$ $\mathrm{pg} \mathrm{m}^{-3}, 19.9-168 \mathrm{pg} \mathrm{m}^{-3}, 7.51-55.6 \mathrm{pg} \mathrm{m}^{-3}$ and $0.33-43.6 \mathrm{pg} \mathrm{m}^{-3}$, respectively. Compared with other researches [24,30], the concentrations in our study were generally higher than those found in the marine atmosphere of the North Sea $\left(110-1400 \mathrm{pg} \mathrm{m}^{-3}\right)$, but several times lower than that in office air particulate matter in China (5.00-148 $\mathrm{ng} \mathrm{m}^{-3}$ ). Marklund et al. [31,32] found that traffic is a major source of OPEs in outdoor environment. While indoor concentrations are typically higher up to several orders of magnitude, due to the emissions from furniture, consumer products and building materials.

In regard to phase distribution, the OPEs found in the active samples mainly accumulated in the airborne particles, which represented $91 \pm 8 \%$ of the total concentration (Fig. 2). In detail, individual particulate associated fractions ranged from < MQL-100\% for TEP, $97-100 \%$ for TCEP, $81-100 \%$ for TCPP, $67-98 \%$ for TBP, $40-$ $100 \%$ for TPhP, $77-100 \%$ for CDP, $85-100 \%$ for TCrP and $100 \%$ for TDCP, respectively. The result is comparable with Moller's study that a mean particulate percentage of $86 \pm 25 \%$ was detected for total atmospheric organophosphate compounds in the North Sea [30]. Also, in the study sampling indoor air at two different flow rates using a personal active sampler, $>99 \%$ of all the organophosphates were distributed on the filters, with less than $1 \%$ found in the PUFs [7].

\subsection{Uptake profiles of PUF-PAS and SIP-PAS disks}

The distribution patterns of atmospheric target compounds could also be derived from the total accumulated amounts on PAS disks at the end of deployment time (Table S4). It was obvious that TCPP represented $87 \%$ and $94 \%$ in PUF-PAS and SIP-PAS, respectively, followed by TCEP (7\% and 3\%, respectively), and TPhP (3\% and $1 \%$, respectively). This was in good agreement with the composition of individual OPEs measured by HV-AAS.

As the concentration levels differed greatly for various OPE compounds, the relative accumulated amount was used in comparison of the uptake profiles over the deployment time (Fig. 3). It

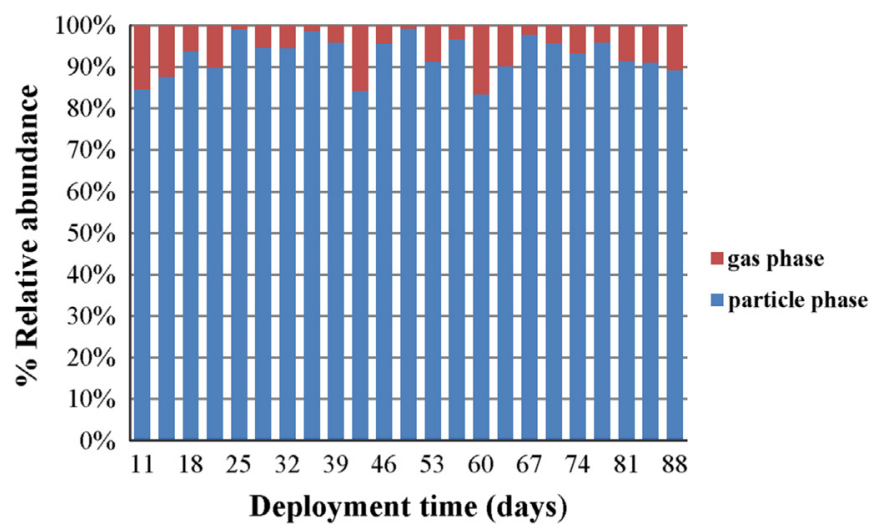

Fig. 2. Distribution of target OPEs in both gas and particle phases in the ambient air during deployment days measured by HV-AAS. could be found that chemicals increased with varying rates and the uptakes were still in linear or curvilinear stages until the end of the period lasting 84 days. For TCPP, the concentration of which was almost one or two orders of magnitudes higher than those of other analogues, a total of $2.30 \mathrm{mg}$ was adsorbed on the SIP disk, while PUF disk absorbed $0.92 \mathrm{mg}$ of analyte at the end of deployment. Moreover, the accumulated amount were 68.3 and $67.4 \mathrm{ng} \mathrm{disk}^{-1}$ for TCEP, 8.00 and $6.60 \mathrm{ng} \mathrm{disk}^{-1}$ for TDCP, 29.8 and $30.7 \mathrm{ng} \mathrm{disk}^{-1}$ for TPhP, 6.20 and $6.70 \mathrm{ng} \mathrm{disk}^{-1}$ for CDP, 13.7 and $13.8 \mathrm{ng} \mathrm{disk}^{-1}$ for TCrP on PUF-PAS and SIP-PAS, respectively. TBP and TEP were not further discussed, because their concentrations varied greatly with lots of outliers, perhaps mainly caused by quite low residue amounts.

From the above theory of PAS, the concentrations and calculation results of various parameters for PUF-PAS and SIP-PAS could be derived (Table 1). As most OPEs in the PAS did not equilibrate, $K_{\text {PSM-A }}$ was underestimated as the lower bound. For TEP, $K_{\text {PSM-A }}$ increased most distinctly from $1.15 \times 10^{6}$ for PUF to $10.9 \times 10^{6}$ for SIP, and the raised value of partition coefficient might suggest $\mathrm{XAD}-4$ resin could absorb much more analyte onto the sampling medium from air.

Calculation of sampling rate should include at least 3 data points within the linear region. As a result, the sampling rates of TCPP were $3.9 \mathrm{~m}^{3} \mathrm{~d}^{-1}$ for PUF-PAS and $6.1 \mathrm{~m}^{3} \mathrm{~d}^{-1}$ for SIP-PAS. And the calculated values were almost the same respectively between the two kinds of PAS disks for other OPE analogues. For instance, the sampling rates were 4.3 and $4.2 \mathrm{~m}^{3} \mathrm{~d}^{-1}$ for TCEP, 2.2 and $2.3 \mathrm{~m}^{3} \mathrm{~d}^{-1}$ for TPhP, 3.7 and $3.8 \mathrm{~m}^{3} \mathrm{~d}^{-1}$ for CDP, and 3.2 and $3.1 \mathrm{~m}^{3} \mathrm{~d}^{-1}$ for TCrP for PUF-disk and SIP-disk, respectively. Though it is expected that uptake is air-side controlled and hence $R$-values could be the same at initial time in both two kinds of PAS disks, discrepant of sampling rates of PUF and SIP disks were reported, for example, for per- and polyfluoroalkyl substances (PFASs) [17,22]. The higher value for TCPP in SIP-PAS might be caused by long harvest intervals. Previous studies indicated that sorption of SIP disks depended on both PUF and XAD powder, and the point where kinetics mechanism switches from initial air-side controlled to subsequent sorbent-side controlled remains to be investigated [22].

In general, the average R-values calculated in this study were $3.3 \pm 1.1$ and $3.5 \pm 1.7 \mathrm{~m}^{3} \mathrm{~d}^{-1}$ for PUF-PAS and SIP-PAS disks in this study, indicating uptake characteristics of the two kinds of PAS disks were similar. To assess the accuracy of our method, peerreviewed data in the literature could be used [33]. The passive sampling rate of PBDEs had been determined as $2.5 \mathrm{~m}^{3} \mathrm{~d}^{-1}$ from the indoor air studies [27]. The suggested value of $4 \mathrm{~m}^{3} \mathrm{~d}^{-1}$ was reported for various kinds of POPs [20], which was in good agreement with our data.

\subsection{Loss of depuration compounds}

Sampling rate, or $R$-value, could also be determined by measuring loss rate of depuration compounds in the passive samplers. In this study, $\mathrm{D}_{27}-\mathrm{TBP}$ and $\mathrm{D}_{15}$-TPhP (20.0 ng each) were spiked into each PAS disk before deployment. The isotopic-labeled compounds will transfer into the atmosphere during the sampling period. And the lost amount, which depends on the physicochemical properties of chemicals and experimental parameters such as wind speed and exposure time, will only due to volatilization instead of degradation [34,35]. Similar to air-side controlled uptake of PAS, the loss according to the partitioning properties is also assumed as air-side controlled. In addition, the loss rate is negatively correlated with the thickness of laminar boundary layer. The thickness would decrease under higher wind speeds, which could lead to faster mass transfer [36,37]. Moecoel [38] reported that uptake rates $\mathrm{R}$ from the loss of DCs showed a 

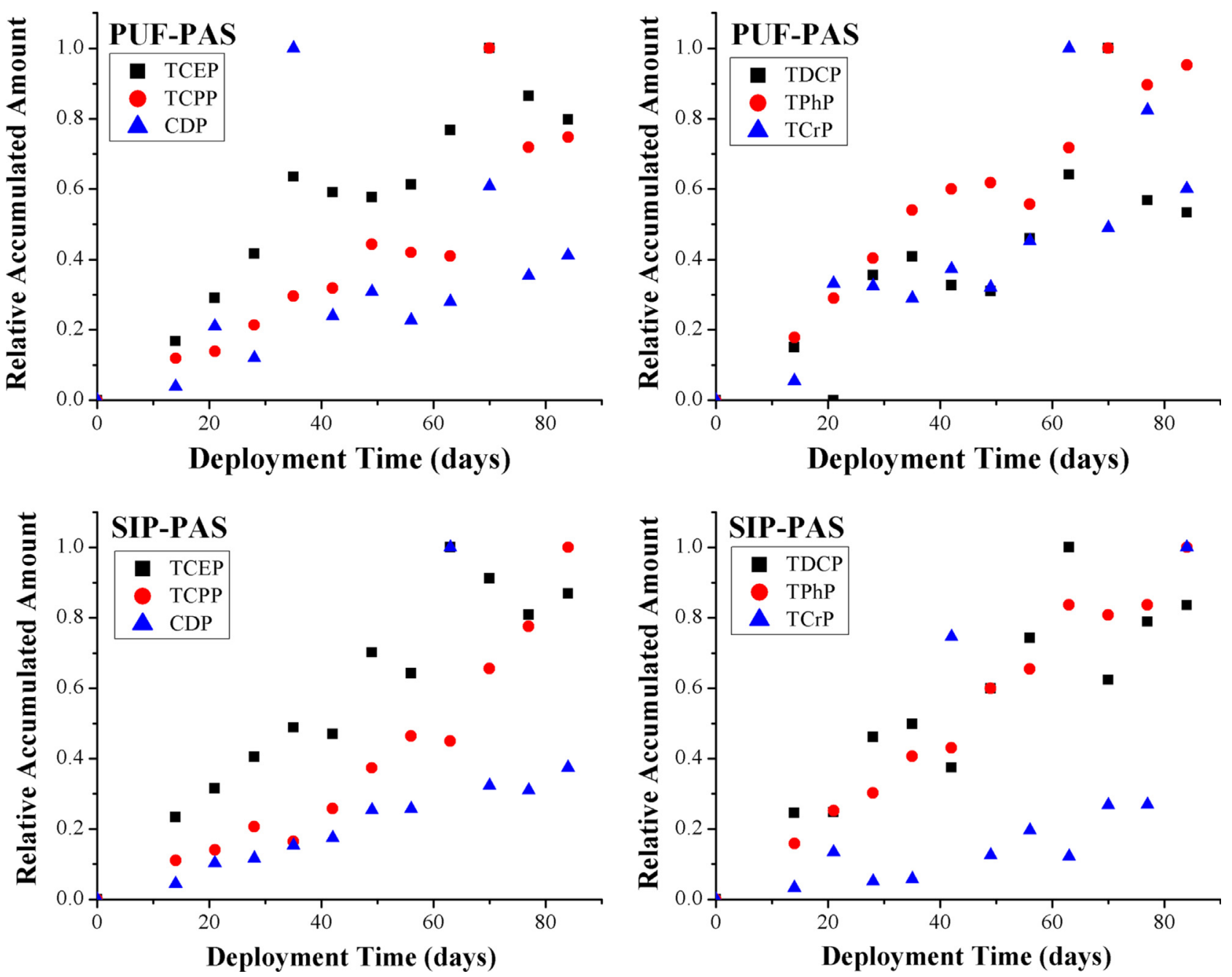

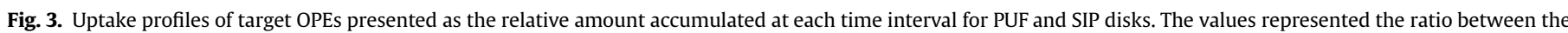
actual amount and the maximum one (including the apparent maximum outlier).

Table 1

Calibration Results for PUF and SIP disks.

\begin{tabular}{|c|c|c|c|c|c|c|c|}
\hline & $c_{\mathrm{A}}\left(\mathrm{pg} \mathrm{m}^{-3}\right)$ & $c_{\mathrm{PUF}}\left(\mathrm{pg} \operatorname{disk}^{-1}\right)$ & $c_{\mathrm{PUF}}\left(\mathrm{pg} \mathrm{m}^{-3}\right.$ disk $)$ & $K_{\text {PUF-Ab }}$ & $\log K_{\text {PUF-A }}$ & $k_{\mathrm{A}}\left(\mathbf{m ~ d}^{-1}\right)$ & $R\left(\mathbf{m}^{-3} \mathbf{d}^{-1}\right)$ \\
\hline \multicolumn{8}{|c|}{ PUF-PAS disk } \\
\hline TCEP & 270 & 68,300 & $3.29 \times 10^{8}$ & $1.22 \times 10^{6}$ & 6.09 & 118 & 4.3 \\
\hline ТСРP & 2537 & 916,000 & $4.43 \times 10^{9}$ & $1.74 \times 10^{6}$ & 6.24 & 107 & 3.9 \\
\hline TEP & 11 & 2620 & $1.27 \times 10^{7}$ & $1.15 \times 10^{6}$ & 6.06 & $\mathrm{nc}^{\mathrm{a}}$ & $\mathrm{nc}^{\mathrm{a}}$ \\
\hline TDCP & 60 & 8000 & $3.86 \times 10^{7}$ & $6.44 \times 10^{5}$ & 5.81 & 74 & 2.7 \\
\hline TBP & 118 & 4000 & $1.93 \times 10^{7}$ & $1.64 \times 10^{5}$ & 5.21 & $\mathrm{nc}^{\mathrm{a}}$ & $\mathrm{nc}^{\mathrm{a}}$ \\
\hline TPhP & 177 & 29,800 & $1.44 \times 10^{8}$ & $8.13 \times 10^{5}$ & 5.91 & 60 & 2.2 \\
\hline CDP & 21 & 6200 & $3.00 \times 10^{7}$ & $1.42 \times 10^{6}$ & 6.15 & 101 & 3.7 \\
\hline $\mathrm{TCrP}$ & 55 & 13,700 & $6.62 \times 10^{7}$ & $1.20 \times 10^{6}$ & 6.08 & 88 & 3.2 \\
\hline \multicolumn{8}{|c|}{ SIP-PAS disk } \\
\hline TCEP & 270 & 67,400 & $3.25 \times 10^{8}$ & $1.21 \times 10^{6}$ & 6.08 & 115 & 4.2 \\
\hline ТСРР & 2537 & $2,299,000$ & $1.11 \times 10^{10}$ & $4.38 \times 10^{6}$ & 6.64 & 167 & 6.1 \\
\hline TEP & 11 & 24,800 & $1.20 \times 10^{8}$ & $1.09 \times 10^{7}$ & 7.04 & $\mathrm{nc}^{\mathrm{a}}$ & $\mathrm{nc}^{\mathrm{a}}$ \\
\hline TDCP & 60 & 6600 & $3.19 \times 10^{7}$ & $5.31 \times 10^{5}$ & 5.73 & 49 & 1.8 \\
\hline ТВP & 118 & 8700 & $4.20 \times 10^{7}$ & $3.56 \times 10^{5}$ & 5.55 & $\mathrm{nc}^{\mathrm{a}}$ & $n c^{\mathrm{a}}$ \\
\hline TPhP & 177 & 30,700 & $1.48 \times 10^{8}$ & $8.38 \times 10^{5}$ & 5.92 & 63 & 2.3 \\
\hline CDP & 21 & 6700 & $3.24 \times 10^{7}$ & $1.54 \times 10^{6}$ & 6.19 & 104 & 3.8 \\
\hline $\mathrm{TCrP}$ & 55 & 13,800 & $6.67 \times 10^{7}$ & $1.21 \times 10^{6}$ & 6.08 & 85 & 3.1 \\
\hline
\end{tabular}

${ }^{\mathrm{b}} \mathrm{PAS}$ disks did not reach equilibrium over the course of the experiment; values were taken at the end of the deployment time to calculate the lower limit of $\mathrm{K}_{\mathrm{PSM}-\mathrm{A} \text {. }}$ ${ }^{\mathrm{a}} \mathrm{nc}=$ not calculable, because of insufficient points. 


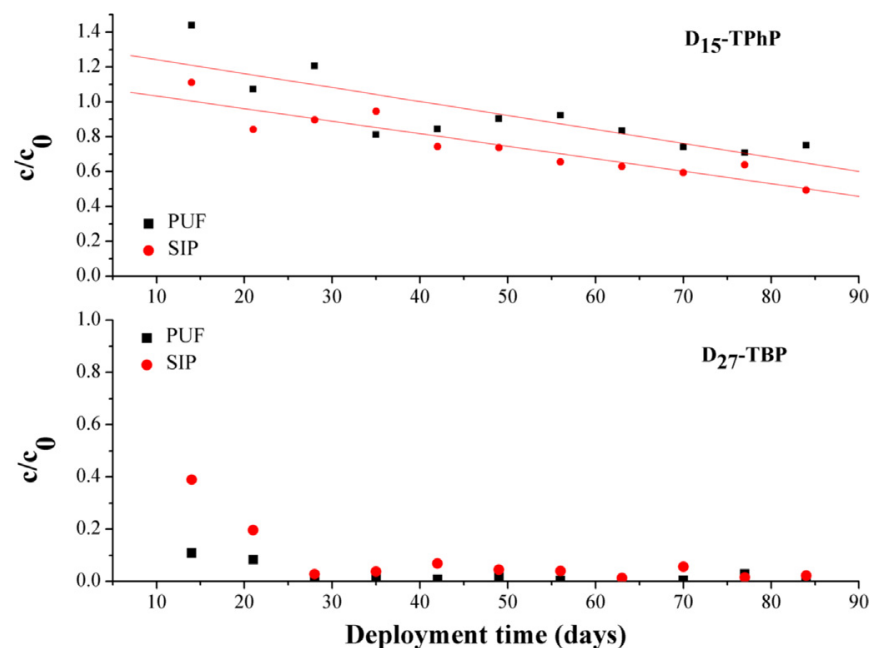

Fig. 4. Loss of DCs during deployment days as fraction ratio of the starting amount. The concentration of the chemical in the PSM at each deployment time $(c)$ is divided by the initial concentration of the chemical at the deployment time $t=0\left(c_{0}\right)$.

significant positive correlation with wind speed.

On assumption that the mass transfer coefficient through the boundary layer of DCs is independent from their $K_{\text {PAS-A }}$, the uptake rate $R$ could be calculated with the following equation:

$R=\frac{-\ln \left(\frac{C_{\mathrm{DC}}}{C_{\mathrm{DC}, 0}}\right) \times K_{\mathrm{PAS}-\mathrm{A}} \times V}{t}$

where $C_{\mathrm{DC}, 0}$ and $C_{\mathrm{DC}}$ are the initial and final concentrations of the DC, $K_{\text {PAS-A }}$ is the PAS-air partition coefficient of the chemical, and $V$ is the volume $\left(\mathrm{m}^{3}\right)$ of PAS. It is necessary to note that the losses of DCs should be in the range of $20-80 \%$, in order to minimize the impacts of analytical variability [38].

As shown in Fig. 4, the two DC compounds showed distinct retention behaviors in the PAS samplers. For $D_{27}$-TBP, almost $90 \%$ and $60 \%$ were lost in PUF-PAS and SIP-PAS in the first 14 days, and the substantial loss up to $97 \%$ was observed in both disks within 30 days. The result could indicate high volatility property of the $\mathrm{D}_{27}$-TBP, which might not be used as a suitable DC. Nevertheless, the residue amount of $\mathrm{D}_{15}$-TPhP in PAS disks showed linear decrease during the sampling period, and little difference was observed between the two PAS types. The loss was $~ 67 \%$ for the PUFPAS and $60 \%$ for the SIP-PAS over the deployment days. As a result, the R-values of $\mathrm{D}_{15}-\mathrm{TPhP}$ were determined as $1.5 \mathrm{~m}^{3} \mathrm{~d}^{-1}$ and $1.7 \mathrm{~m}^{3} \mathrm{~d}^{-1}$ respectively for PUF-PAS and SIP-PAS. It is a little lower than the $R$-value ( 2.2 and $2.3 \mathrm{~m}^{3} \mathrm{~d}^{-1}$ ) calculated in our uptake study using native compounds. It might be related with the lower values of estimated $K_{\mathrm{PSM}-\mathrm{A}}$ by the DC calculation method. However, it was discussed that the rates between the uptake of native chemical and the loss of DC might not be necessarily equal because of inhomogeneities in diffusivities, as a kinetic resistance might exist during chemical transfer into the PSM [39].

\subsection{Calculation of equivalent sampling air volume}

The equivalent sampling air volume $\left(V_{\mathrm{AIR}}\right)$ could be described by combining Eq. (1) and Eq. (3), where

$V_{\mathrm{AIR}}=k_{\mathrm{PSM}-\mathrm{A}} \times V_{\mathrm{PSM}} \times\left\{1-\exp -\left[\left(\frac{A_{\mathrm{PSM}}}{V_{\mathrm{PSM}}} \times \frac{k_{\mathrm{A}}}{k_{\mathrm{PSM}}}\right) \times t\right]\right\}$

Fig. 5 is the calibrated plot of $V_{\text {AIR }}$ along with deployment time in PUF disks, using parameters of $k_{\mathrm{A}}$ and $\mathrm{K}_{\mathrm{PUF}-\mathrm{A}}$ values in Table 1. In

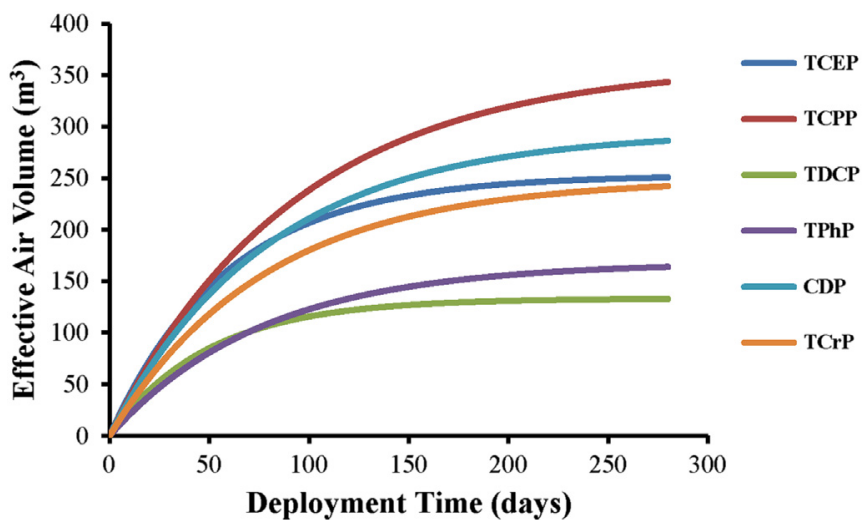

Fig. 5. Uptake profiles of PUF-PAS for TCEP, TCPP, TDCP, TPhP, CDP, TCrP derived using Eq. (4) and uptake parameters listed in Table 1. Time is extended to exceed $t_{95}$ for target analytes.

order to determine the extent of the linear and equilibrium uptake phase of the plot, the constant $k_{\mathrm{U}}$ was utilized [28], where

$k_{U}=\left(A_{\mathrm{PSM}} / V_{\mathrm{PSM}}\right)\left(k_{\mathrm{A}} / k_{\mathrm{PSM}-\mathrm{A}}\right)$

when the disks accumulated $25 \%$ and $95 \%$ of the equilibrium value, $t_{25}=\ln (0.75) / k_{\mathrm{U}}$ and $t_{95}=\ln (0.05) / k_{\mathrm{U}}$ were used to calculate the corresponding deployment time of PAS. As a result, the predicted $t_{25}$ of TPhP, CDP and TCrP lasted about 3 weeks in both PAS types, while the linear time was about 2 weeks for TCEP and TDCP. However, difference on sorption capacities between the two media existed for TCPP, as SIP-PAS had longer linear phase up to 43 days compared with 27 days of PUF-PAS (Table S5). Besides, $t_{95}$ of all the investigated chemicals in our work ranged from 6 to 15 months, indicating that the deployment in our study might not have reached equilibrium. Similar results were found in SIP-PAS sampling of PFASs that $t_{95}$ ranged from 9 months up to 32 months with similar sampling rates $\left(2.9-4.4 \mathrm{~m}^{3} \mathrm{~d}^{-1}\right)$, which implies more amount of PFASs could accumulate on the disks than those of OPEs [17].

\subsection{Uptake performances of two passive sampling disks}

The uptake performances of PUF-PAS and SIP-PAS for accumulating OPEs were compared and the retention behaviors varied for different chemicals. The presence of XAD powder in SIP-PAS led to higher sorption capacity and longer period of linear-phase sampling for more volatile chemicals [17,22]. In our study, greater sorption capacities were observed for TCPP, TEP and TBP. As the most abundant chemical, TCPP concentrated on SIP disks as much as 2.5 times compared with PUF. With regards to the most volatile compounds in this study, the total amounts of TBP were $4.00 \mathrm{ng} \mathrm{disk}{ }^{-1}$ on PUF disks and $8.70 \mathrm{ng}$ disk $^{-1}$ on SIP disks at the end of whole sampling periods. Distinct behaviors were also observed that $2.62 \mathrm{ng}$ and $24.8 \mathrm{ng}$ of TEP accumulated on PUF-PAS and SIP-PAS, respectively. SIP-PAS disks were found with improved adsorption capacity towards volatile chemicals due to its larger surface area [30]. Thus, the results might also suggest the positive impact of impregnated XAD-4 material on the adsorption of OPE analytes in the atmosphere. On the other hand, only minor difference was obtained between the two mediums on both adsorption capacities and sampling rates for other OPE chemicals (Table 1).

\section{Conclusions}

In this study, a robust passive air sampling method for 
organophosphate esters in the ambient air was developed. Contamination levels of eight analytes were investigated by HV-AAS and PAS techniques. OPE residue concentrations and compositions measured by PAS were comparable to those of HV-AAS, suggesting passive air samplers could be used as an alternative to more frequently used active samplers. Adsorption abilities of both PUF-PAS and SIP-PAS disks were examined. SIP-PAS disk could provide longer linear-phase sampling period and higher sorption capacity for more volatile homologues. While, similar sorption abilities were observed for other OPE chemicals by both PUF-PAS and SIPPAS disks. The calibrated parameters and sampling plots were also supplied in our study, which could be referenced in the following researches of OPEs on a broader scale.

\section{Acknowledgments}

This work was jointly supported by the High-Tech Research and Development Program of China (2013AA065201), the National Natural Science Foundation of China (21207140 and 21461142001) and Youth Innovation Promotion Association CAS projects.

\section{Appendix A. Supplementary materials}

Supplementary data associated with this article can be found in the online version at http://dx.doi.org/10.1016/j.talanta.2015.09. 034.

\section{References}

[1] A. Moller, R. Sturm, Z. Xie, M. Cai, J. He, R. Ebinghaus, Environ. Sci. Technol. 46 (2012) 3127-3134.

[2] A. Marklund, B. Andersson, P. Haglund, Chemosphere 53 (2003) 1137-1146.

[3] S.H. Brandsma, J. de Boer, P.E.G. Leonards, W.P. Cofino, A. Covaci, P.E. G. Leonards, TrAC Trends Anal. Chem. 43 (2013) 217-228.

[4] A. Meijers, R.C. Van der Leer, Water Res. 10 (1976) 597-604.

[5] J. Castro-Jimenez, N. Berrojalbiz, M. Pizarro, J. Dachs, Environ. Sci. Technol. 48 (2014) 3203-3209.

[6] T. Reemtsma, J.B. Quintana, R. Rodil, M. García-López, I. Rodríguez, TrAC Trends Anal. Chem. 27 (2008) 727-737.

[7] H. Carlsson, U. Nilsson, G. Becker, C. Östman, Environ. Sci. Technol. 31 (1997) $2931-2936$.

[8] I. van der Veen, J. de Boer, Chemosphere 88 (2012) 1119-1153.
[9] M. Wensing, E. Uhde, T. Salthammer, Sci. Total Environ. 339 (2005) 19-40.

[10] J.G. Camarasa, E. Serra-Baldrich, Contact Dermat. 26 (1992) 264-265.

[11] W.H. Organization, Triphenyl phosphate, 1991. 〈http://www.who.int/iris/han dle/10665/ 40299>.

[12] W.H. Organization, Tri-n-butyl phosphate, 1991. 〈http://www.who.int/iris/ handle/ 10665/39857>.

[13] H. Matthews, S. Eustis, J. Haseman, Toxicol. Sci. 20 (1993) 477-485.

[14] T. Sato, K. Watanabe, H. Nagase, H. Kito, M. Niikawa, Y. Yoshioka, Toxicol. Environ. Chem. 59 (1997) 305-313.

[15] N. Van den Eede, A.C. Dirtu, H. Neels, A. Covaci, Environ. Int. 37 (2011) $454-461$.

[16] M.S. Mäkinen, M.R. Mäkinen, J.T. Koistinen, A.L. Pasanen, P.O. Pasanen, P.J. Kalliokoski, A.M. Korpi, Environ. Sci. Technol. 43 (2009) 941-947.

[17] L. Ahrens, T. Harner, M. Shoeib, M. Koblizkova, E.J. Reiner, Environ. Sci. Technol. 47 (2013) 14024-14033.

[18] T. Harner, N.J. Farrar, M. Shoeib, K.C. Jones, F.A. Gobas, Environ. Sci. Technol. 37 (2003) 2486-2493.

[19] K. Pozo, T. Harner, F. Wania, D.C. Muir, K.C. Jones, L.A. Barrie, Environ. Sci. Technol. 40 (2006) 4867-4873.

[20] K. Pozo, T. Harner, S.C. Lee, F. Wania, D.C. Muir, K.C. Jones, Environ. Sci. Technol. 43 (2008) 796-803.

[21] F.M. Jaward, N.J. Farrar, T. Harner, A.J. Sweetman, K.C. Jones, Environ. Sci. Technol. 38 (2004) 34-41.

[22] M. Shoeib, T. Harner, S.C. Lee, D. Lane, J. Zhu, Anal. Chem. 80 (2008) 675-682.

[23] S. Genualdi, S.C. Lee, M. Shoeib, A. Gawor, L. Ahrens, T. Harner, Environ. Sci. Technol. 44 (2010) 5534-5539.

[24] F. Yang, J. Ding, W. Huang, W. Xie, W. Liu, Environ. Sci. Technol. 48 (2014) 63-70.

[25] S. Lai, Z. Xie, T. Song, J. Tang, Y. Zhang, W. Mi, J. Peng, Y. Zhao, S. Zou, R. Ebinghaus, Chemosphere 127 (2015) 195-200.

[26] Y. Ni, K. Kumagai, Y. Yanagisawa, Atmos. Environ. 41 (2007) 3235-3240.

[27] B.H. Wilford, T. Harner, J. Zhu, M. Shoeib, K.C. Jones, Environ. Sci. Technol. 38 (2004) 5312-5318.

[28] M. Shoeib, T. Harner, Environ. Sci. Technol. 36 (2002) 4142-4151.

[29] M.E. Bartkow, K. Booij, K.E. Kennedy, J.F. Müller, D.W. Hawker, Chemosphere 60 (2005) 170-176.

[30] A. Möller, Z. Xie, A. Caba, R. Sturm, R. Ebinghaus, Environ. Pollut. 159 (2011) 3660-3665.

[31] A. Marklund, B. Andersson, P. Haglund, Environ. Sci. Technol. 39 (2005) 3555-3562.

[32] A. Marklund, B. Andersson, P. Haglund, J. Environ. Monit. 7 (2005) 814-819.

[33] H. Altundag, M.S. Dundar, C.S. Keskin, Ecol. Chem. Eng. S 20 (2013) 257-264.

[34] J.N. Huckins, J.D. Petty, J.A. Lebo, F.V. Almeida, K. Booij, D.A. Alvarez, W. L. Cranor, R.C. Clark, B.B. Mogensen, Environ. Sci. Technol. 36 (2002) 85-91.

[35] K. Pozo, T. Harner, M. Shoeib, R. Urrutia, R. Barra, O. Parra, S. Focardi, Environ. Sci. Technol. 38 (2004) 6529-6537.

[36] J. Klánová, P. Èupr, Ji Kohoutek, T. Harner, Environ. Sci. Technol. 42 (2007) $550-555$.

[37] T. Gouin, T. Harner, P. Blanchard, D. Mackay, Environ. Sci. Technol. 39 (2005) 9115-9122.

[38] C. Moeckel, T. Harner, L. Nizzetto, B. Strandberg, A. Lindroth, K.C. Jones, Environ. Sci. Technol. 43 (2009) 3227-3232.

[39] X. Zhang, M. Tsurukawa, T. Nakano, Y.D. Lei, F. Wania, Environ. Sci. Technol. 45 (2011) 10509-10515. 